\section{Kink Drift in Oscillating Fields}

Recently, Sukstanskii and Primak [1] investigated drift motion of kinks in the $\phi^{4}$ model induced by oscillating external fields. They applied a standard adiabatic perturbation theory for solitons and calculated the nonlinear mobility of a $\phi^{4}$ kink. The aim of our Comment is twofold. First, we demonstrate that the phenomenon of kink drift is a trivial consequence of the theory of averaged soliton dynamics in rapidly oscillating fields developed for the $\phi^{4}$ model in our earlier paper [2] (see also [3] for other examples). Indeed, due to the action of two oscillating fields as in the case considered in [1], the effective potential supporting averaged kink dynamics becomes asymmetric, and this is the physical reason for kink drift. Second, direct application of our theory [2] to the problem considered in Ref. [1] demonstrates that the adiabatic approximation used in [1] is likely to be invalid for the case of oscillating fields: We show that, for large values of the field frequency $\omega$, the velocity of the kink drift $V_{\mathrm{dr}}$ decays as $\omega^{-2}$, instead of the result $V_{\mathrm{dr}} \propto \omega^{-4}$ predicted by Eq. (17) of Ref. [1]. As we will see below, our prediction is in very good agreement with direct numerical integration of the full perturbed $\phi^{4}$ equation.

According to Ref. [1], we consider the $\phi^{4}$ model with two monochromatic fields of frequency $\omega$ and with arbitrary phase shift $\chi$,

$$
\phi_{t t}-\phi_{x x}-\phi+\phi^{3}=h_{1}(t)+h_{2}(t) \phi-\gamma \phi_{t},
$$

where $h_{1}(t)=h_{10} \cos \omega t, h_{2}(t)=h_{20} \cos (\omega t+\chi)$, $\gamma$ is a dissipative constant, and dimensionless units have been used. Because the frequency $\omega$ is assumed to be of order (or larger) of the system eigenfrequency, the adiabatic approximation cannot be used to describe the effect of oscillating perturbations on the kink dynamics. Instead, we follow the approach developed in Refs. [2,3] and decompose the wave field $\phi$ into a sum of slow and fast varying parts, $\phi=\Phi+\xi$, deriving the equation for the slowly varying field $\Phi$ (see [2]),

$$
\Phi_{t t}-\Phi_{x x}-\Phi+\Phi^{3}+3 \Phi\left\langle\xi^{2}\right\rangle=\left\langle h_{2}(t) \xi\right\rangle-\gamma \Phi_{t} .
$$

This approach is at least valid for $\omega>1$, which is consistent with the fact that we are mainly interested in the large $\omega$ asymptotics of this problem. Similar to Ref. [2], the fast varying part of the solution can be easily found as $\xi(t)=A \cos \omega t+B \sin \omega t$, where

$$
\begin{aligned}
& A=\frac{1}{\omega^{2}+\gamma^{2}}\left\{\frac{1}{2} h_{10}+h_{20}\left[\cos \chi-\frac{\gamma}{\omega} \sin \chi\right] \Phi\right\}, \\
& B=\frac{1}{\omega^{2}+\gamma^{2}}\left\{\frac{\gamma}{2 \omega} h_{10}+h_{20}\left[\frac{\gamma}{\omega} \cos \chi+\sin \chi\right] \Phi\right\} .
\end{aligned}
$$

The averaged equation derived above completely solves the problem of kink drift because it immediately shows that simultaneous action of two oscillating fields forces the effective potential of the averaged $\phi^{4}$ model to be asymmetric, and therefore it creates an effective force for

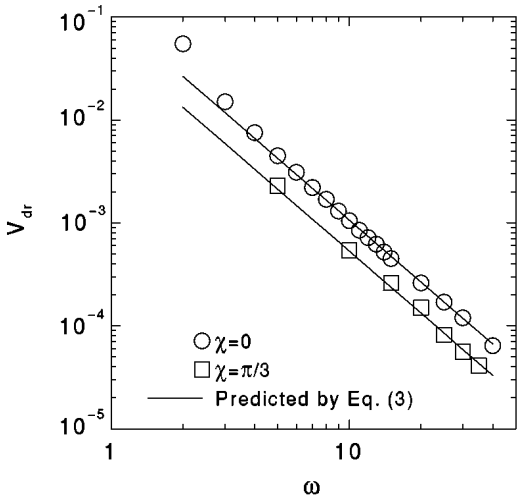

FIG. 1. Log-log plot of the drift velocity vs the driving frequency for $h_{10}=h_{20}=\gamma=0.1$, and $\chi=0(\bigcirc), \chi=\pi / 3$ ( $\square$ ).

a translational motion of a kink. This simple physics has not been noticed in Ref. [1].

Moreover, in the limit of large $\omega$, i.e., $\omega^{2} \gg \gamma^{2}$, we easily obtain $\Phi_{t t}-\Phi_{x x}-\Phi_{m}^{2} \Phi+\Phi^{3}=F-\gamma \Phi_{t}$, where $\Phi_{m}^{2}=1-h_{20}^{2} / 2 \omega^{2}$ and $F=\left(h_{10} h_{20} / 2 \omega^{2}\right) \cos \chi$. Considering now a kink in the form $\Phi= \pm \Phi_{m} \times$ $\tanh \left[\Phi_{m}\left(x-x_{0}\right) / \sqrt{2}\right]$, we find that, under the action of the effective force $F$, it starts to move with the drift velocity

$$
\dot{x}_{0}=V_{\mathrm{dr}}= \pm \frac{3}{2 \sqrt{2}} \frac{h_{10} h_{20} \cos \chi}{\gamma \omega^{2}} .
$$

The result (2) predicts that, for large $\omega$, the drift velocity $V_{\mathrm{dr}}$ decays as $\omega^{-2}$ unlike the result $V_{\mathrm{dr}} \propto \omega^{-4}$ which follows from the application of the adiabatic perturbation theory in Ref. [1]. Figure 1 illustrates the great accuracy of our prediction (2) as compared to numerical results on the full perturbed $\phi^{4}$ equation (see [2] for details on the numerical procedure).

A.S. is supported by CICyT (Spain), Grant No. MAT95-0325.

Yuri S. Kivshar

Optical Sciences Centre

The Australian National University

ACT 0200 Canberra, Australia

Angel Sánchez

Departamento de Matematicas

Escuela Politecnica Superior

Universidad Carlos III de Madrid

E-28911 Leganes Madrid, Spain

Received 3 January 1996

PACS numbers: 03.50.Kk

[S0031-9007(96)00650-3]

[1] A.L. Sukstanskii and K. I. Primak, Phys. Rev. Lett. 75, 3029 (1995).

[2] Yu. S. Kivshar, A. Sánchez, and L. Vázquez, Phys. Rev. A 45, 1207 (1992).

[3] Yu. S. Kivshar, N. Grønbech-Jensen, and R. D. Parmentier, Phys. Rev. E 49, 4542 (1994). 